\title{
Singlet Fission and Triplet Transfer to PbS Quantum Dots in TIPS- Tetracene Carboxylic Acid Ligands
}

\author{
Nathaniel J. L. K. Davis ${ }^{a}$, Jesse R. Allardice ${ }^{a}$, James Xiao ${ }^{a}$, Anthony J. Petty II ${ }^{b}$, Neil C. \\ Greenham ${ }^{\mathrm{a}}$, John E. Anthony ${ }^{\mathrm{b}}$ and Akshay Rao ${ }^{\mathrm{a} *}$.
}

\begin{abstract}
Contacts
${ }^{a}$ Cavendish Laboratory, University of Cambridge, J.J. Thomson Avenue, Cambridge, CB3 OHE, UK

${ }^{\mathrm{b}}$ Department of Chemistry, University of Kentucky ,161 Jacobs Science Building, Lexington KY 405060174

*ar525@cam.ac.uk
\end{abstract}




\section{Abstract}

Singlet exciton fission allows for the generation of two triplet excitons for each photon absorbed within an organic semiconductor. Efficient harvesting of these triplets could allow for the Shockley-Queisser limit on the power conversion efficiency of single-junction photovoltaics to be broken. Here, we show that singlet fission molecules bound directly to $\mathrm{PbS}$ quantum dots as ligands can undergo singlet fission with near unity efficiency and can transfer triplets sequentially into the PbS with near unity efficiency. Within the PbS the excitations recombine, giving rise of the emission of photons. This allows for the doubling of the quantum dot photoluminescence quantum efficiency when photons are absorbed by the singlet fission ligand, as compared to when directly absorbed in the quantum dot. Our approach demonstrates that it is possible to convert the exciton multiplication process of singlet fission into a photon multiplication process and provides a new path to harness singlet fission with photovoltaics.

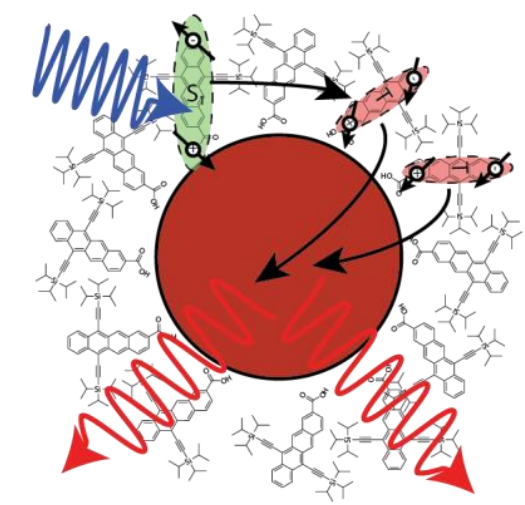

Table of Contents Figure: Singlet fission and triplet transfer to pbs quantum dots in tipstetracene carboxylic acid ligands 
Singlet fission (SF) is an exciton multiplication process which occurs in some organic semiconductors, where the initially photogenerated singlet exciton $\left(\mathrm{S}_{1}\right)$ can convert to an entangled triplet pair (TT state), which then breaks up to form two free triplet excitons $\left(2 \mathrm{~T}_{1}\right)$ on neighbouring chromophores ${ }^{1-3}$. Thus, in an efficient SF material each absorbed photon produces two electron-hole pairs.

SF has attracted great interest recently as a means to circumvent the Shockley-Queisser limit on the power conversion efficiency of single-junction photovoltaics (PVs) ${ }^{4,5}$. This is because SF allows for the generation of two electron-hole pairs from high-energy photons absorbed in the SF material, which is then paired with a low-bandgap material which absorbs lowenergy photons generating one electron-hole pair. Thus, thermalisation losses could be greatly reduced and the efficiency limit on a single-junction PV could be lifted from $33 \%$ to $44 \%$, as has been described in detail elsewhere ${ }^{3,6}$.

SF has been implemented with many types of PV devices and has been demonstrated to enhance photocurrent in organic solar cells ${ }^{7}$, organic/nanocrystal hybrid solar cells ${ }^{8}$ as well as a parallel tandem silicon/pentacene solar cell ${ }^{9}$. However, these designs are limited by the need to dissociate triplet excitons into free electrons and holes, a process that comes with significant losses ${ }^{3}$. Recently, we have proposed an alternative method to harvest SF to enhance PV efficiencies ${ }^{3}$. This proposal is based on the discovery that it is possible to efficiently transfer the energy of triplet excitons into inorganic colloidal quantum dots (QDs), such as $\mathrm{PbS}^{10}$ and $\mathrm{PbSe}^{11}$. This allows the luminescent harvesting of triplet excitons and leads to the concept of the Singlet Fission Photon Multiplier, which consists of a film of SF molecules doped with QDs. Photon absorption leads to the formation of singlet excitons $\left(\mathrm{S}_{1}\right)$ in the SF material which then undergo fission to form free triplets $\left(T_{1}\right)$. These triplets then diffuse within the film until they encounter a $Q D$ into which their energy can be transferred. Once the energy is transferred into the $Q D$, radiative recombination can occur, emitting a lowenergy photon which can then be directed into a conventional inorganic PV, such as Si. Thus, the triplet excitons are radiatively harvested, converting an exciton multiplication process into a photon multiplication process. Full details of the Singlet Fission Photon Multiplier and its advantages over other potential routes to breaking the Shockley-Queisser limit are highlighted in a recent review ${ }^{3}$.

The quantum efficiency of a singlet fission photon multiplication process ( $\eta_{\mathrm{PM}}$ ) is determined by the quantum efficiencies of four steps, the efficiency of SF $\left(\eta_{S F}\right)$, the efficiency of triplet diffusion from the SF material to the QDs vicinity $\left(\eta_{\mathrm{TD}}\right)$, the efficiency of triplet transfer from the SF material into the QDs $\left(\eta_{T T}\right)$ and the efficiency of light emission from the QDs ( $\left.\eta_{P L}\right)$,

$$
\eta_{P M}=\eta_{S F} \cdot \eta_{T D} \cdot \eta_{T T} \cdot \eta_{P L} \text {. }
$$

In the ideal case, $\eta_{\mathrm{PM}}=200 \%$, with each high-energy photon absorbed in the SF material giving rise to the emission of two low-energy photons from the QDs.

The challenge in achieving this goal is to simultaneously maximise the efficiency of each step in the process. For instance, SF yields are known to be highly sensitive to molecular packing ${ }^{12}$, as is triplet diffusion ${ }^{12}$, but these need to be optimised in the presence of the QDs, which might disrupt the packing of the chromophores. Similarly, the triplet excitons need to diffuse 
close $(<1 \mathrm{~nm})$ to the QDs in order to undergo energy transfer into them via short-range Dextertype energy transfer ${ }^{10,13,14}$. So, the interface between the SF molecules and the QD needs to be carefully engineered, yet at the same time the SF molecules near the nanocrystal must still be capable of undergoing SF. Previously, efficient triplet transfer into QDs has been achieved via the use of short-chained ligands such as benzenedithiol ${ }^{10,13,14}$. However, these ligands to do not adequately passivate the QDs surface, leading to poor luminescence efficiency.

Organic semiconductors, such as acenes, have previously been attached directly to QDs to act as triplet acceptors ${ }^{15-17}$. Here, excitation of the QD with low-energy photons produces an exciton in the QD, but does not excite the organic ligand. The energy of the QD is chosen such that it lies above the triplet energy on the organic ligand thus facilitating efficient triplet energy transfer from the QD into the ligand. This strategy has proved very effective in QDorganic hybrid upconversion systems, allowing for the efficient extraction of triplets from QDs via suitable ligands ${ }^{15-19}$.

A similar strategy might be adopted to channel triplets from the organic semiconductor into the QDs, as laid out in Figure 1. However, a priori one would not expect SF materials attached as ligands to QDs to be capable of efficient fission, as it is unlikely that they would adopt the appropriate molecular packing for the efficient formation and subsequent dissociation of TT states, both of which are highly sensitive to intermolecular configuration ${ }^{2,12,20}$. Furthermore, even if two triplet states were formed on the ligands, in order for them to be harvested efficiently they must be injected sequentially into the QD, as the injection of both triplets simultaneously would lead to a biexciton with poor luminescence yield. Hence, the first triplet must be injected into the QD where it recombines radiatively leading to photon emission, while the other triplet must remain on the ligands without recombining non-radiatively or being quenched by surface defects on the QDs. Following the emission of the first photon from the QD the second triplet must be injected into the QD where it can recombine radiatively. At first glance, this would appear to be a very challenging set of requirements to achieve.

Here, we show that SF molecules bound directly to QD as ligands can undergo singlet fission with near unity efficiency and can transfer triplets sequentially into the QD with near unity efficiency. This allows for the doubling of the QD photoluminescence quantum efficiency when photons are absorbed by the singlet fission ligand, as compared to when directly absorbed in the QD.

As a proof of concept experiment we employ a carboxylic-acid-functionalised TIPS-tetracene ligand 6,11-bis((triisopropylsilyl)ethynyl)tetracene-2-carboxylic acid), referred to as TetCAL, attached to PbS QDs. The singlet fission dynamics of TIPS-tetracene have been extensively studied both in solution and in the solid state. Fission in this system is endothermic by around $200-300 \mathrm{meV}$ and the breakup of TT states into free triplets is highly sensitive to crystal packing. The spectroscopic signatures of the various excited states in this system are also well studied $^{1,21}$. Figure 1 illustrates the QD with TetCAL ligands and the various photophysical processes that must occur for efficient harvesting of the singlet fission process. 


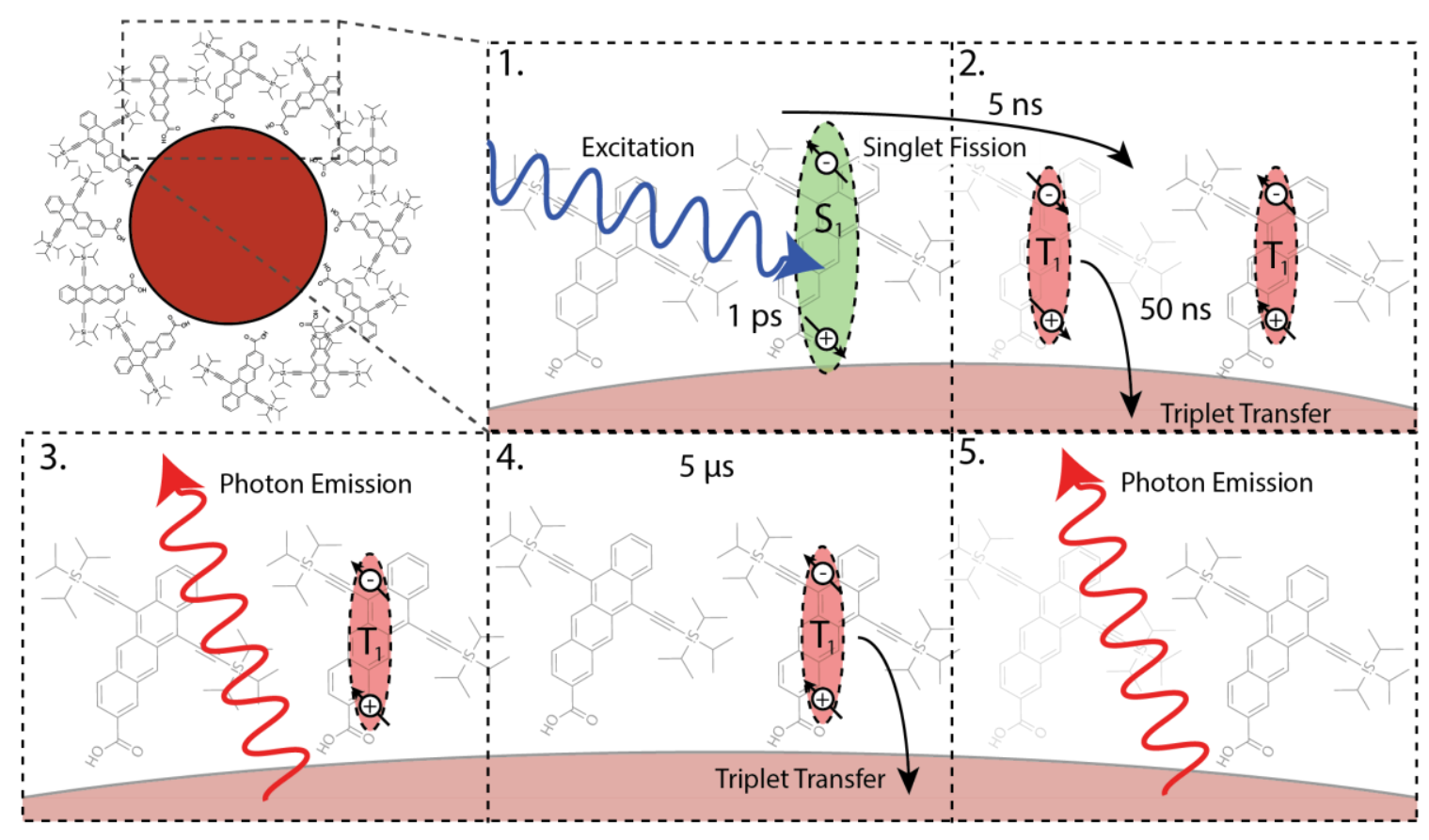

Figure 1: Schematic of the PbS - Tet system with the TetCAL singlet fission ligands on the surface of the PbS QD and energy level diagram of the tetracene/PbS nanocrystal interface. We propose that the system functions as follows: (1) photon absorption leads to the formation of singlet excitons on the ligands ( $\approx 1 \mathrm{ps}),(2)$ singlet fission leads to the formation of triplet excitons on neighbouring ligands $(\approx 5 \mathrm{~ns})$, one of which is then injected to the $\mathrm{PbS}$ $(\approx 50 \mathrm{~ns}),(3)$ the first excitation recombines in the PbS leading to photon emission, $(4)$ the other triplet then transfers into the $\mathrm{PbS}(\approx 5 \mu \mathrm{s})$, and $(5)$ the second excitation recombines in the $\mathrm{PbS}$ leading to photon emission.

Figure $2 \mathrm{a}$ shows the absorption and emission spectra of the PbS QDs with the native oleic acid (referred to as PbS-OA) ligands and those modified with the TetCAL ligand (referred to as PbStet). The PbS QDs have a bandgap of $0.93 \mathrm{eV}$ and show a strong excitonic absorption feature around $1150 \mathrm{~nm}$ with absorption extending into the blue and peak photoluminescence (PL) at $\approx 1275 \mathrm{~nm}$. The native oleic acid ligands were displaced via the addition of TetCAL in toluene as described in the methods section. We note that the purification steps were repeated until there was no free ligand detectable in the washing solution, ensuring that all measured TetCAL was attached to the QDs. The absorption features associated with the TetCAL ligand can clearly be seen at high energies. The absorption spectra of $\mathrm{PbS}-$ tet was found to be a liner combination of the TetCal and the initial PbS QDs spectra, implying no change to the optical properties of the PbS QDs (SI Figure 1). Upon ligand exchange the QDs remain structurally the same, as determined via size distributions measured with transmission electron microscopy (TEM) (SI Figures S2 and S3). Although there is no shift in the PL spectra the photoluminescence quantum efficiency (PLQE) of the QDs (measured with excitation at $785 \mathrm{~nm}$ ) decreases from $35 \%$ to $9 \%$ following the ligand exchange from the native oleic acid ligands to TetCAL ligands. 
QD surface ligand density was calculated using the absorbance spectra of a known concentration of PbS-Tet in toluene and the molar extinction coefficient of TetCAL (SI Figure 4) and found to be 1.06 ligands per $\mathrm{nm}^{2}$, when approximating the QD to the spherical. This is less than the ligand density expected from native oleic acid ligands (OA), which was found to be between 3.0 per $\mathrm{nm}^{2}$ in $\mathrm{PbS} \mathrm{QDs}^{22}$. This lowering of ligand density may result in unpassivated surface sites on the PbS QDs, leading to the observed lowering of PLQE.

Figure $2 \mathrm{~b}$ shows the excitation spectrum of PbS-tet along with its absorption spectrum. The excitation spectrum is measured by monitoring the PL at $1250 \mathrm{~nm}$ while scanning the excitation wavelength. A clear contribution from TetCAL absorption features can be detected at higher wavelengths. This proves that energy absorbed into the TetCAL ligand is transferred into the QDs where it leads to emission.
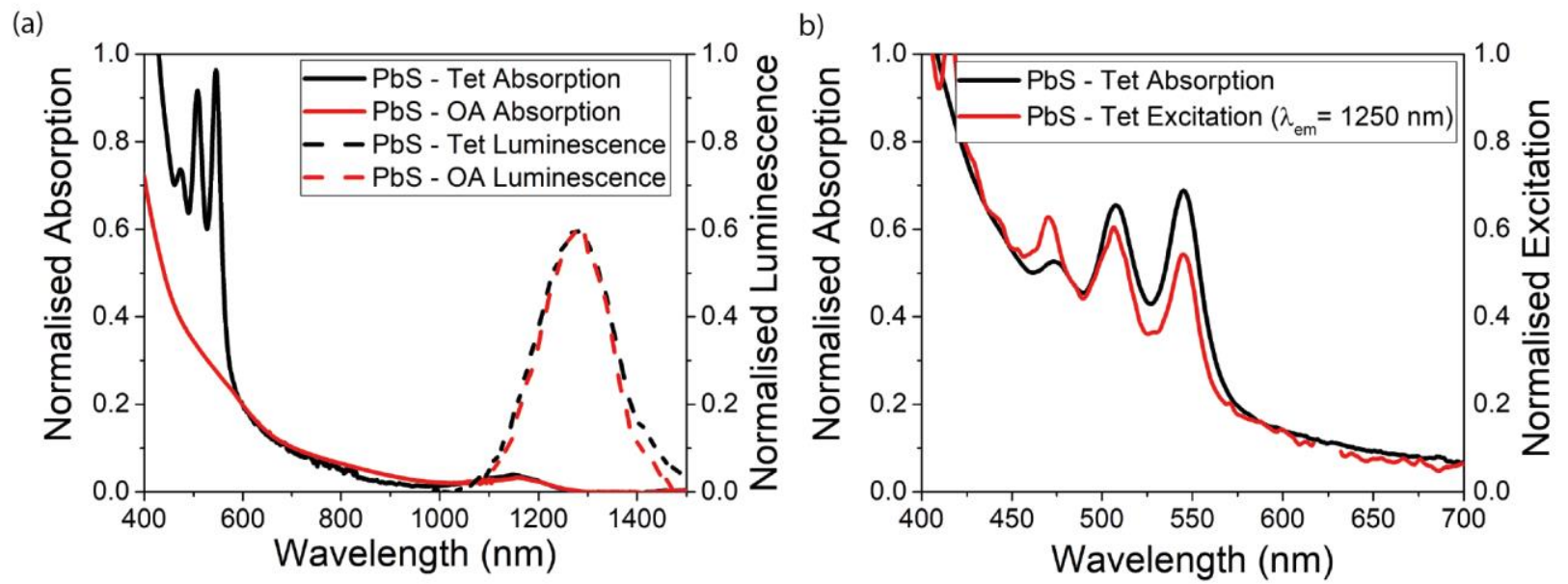

Figure 2: (a) Absorption and photoluminescence spectra of PbS QDs with oleic acid ligands (PbS-OA) and PbS QDs with TetCAL (PbS-Tet). (b) Absorbance and photoluminescence excitation spectra for PbS-Tet. The excitation spectrum is measured at an emission wavelength of $1250 \mathrm{~nm}$. The discontinuity in the PbS - Tet Excitation spectra is a deletion of the signal resulting from the second harmonic interaction of $\approx 625 \mathrm{~nm}$ in our monochromator being registered as $1250 \mathrm{~nm}$ emission.

Figure 3 shows the PLQE of the PbS-tet QDs when excited at $785 \mathrm{~nm}$ and $532 \mathrm{~nm}$. At $785 \mathrm{~nm}$ photons are absorbed only in the PbS and a PLQE of $9 \%$ is recorded. At $532 \mathrm{~nm}$, photons are primarily absorbed into the TetCAL ligand as this corresponds to the peak of ligand absorption (see Figure 2a). The PLQE with $532 \mathrm{~nm}$ excitation is $17 \%$, nearly double that with $785 \mathrm{~nm}$ excitation. We see no fluorescence or phosphoresces from the TetCAL in our systems under $532 \mathrm{~nm}$ excitation (SI Figure 5). The emission of the ligands is completely quenched by the singlet fission process. Unlike solid state films of tetracene based molecules, no delayed PL is observed, showing that triplets cannot fuse back to the emissive singlet. Taken together, this further adds to the evidence for very efficient SF and triplet transfer. As a control, the PLQE of the PbS-OA QDs was measured and found to be $35 \%$ with both $785 \mathrm{~nm}$ and $532 \mathrm{~nm}$ 
excitation (SI Figure 6). The doubling of the PLQE in the PbS-tet QDs when the TetCAL ligands are excited suggests that efficient singlet fission and triplet energy transfer are occurring in this system.

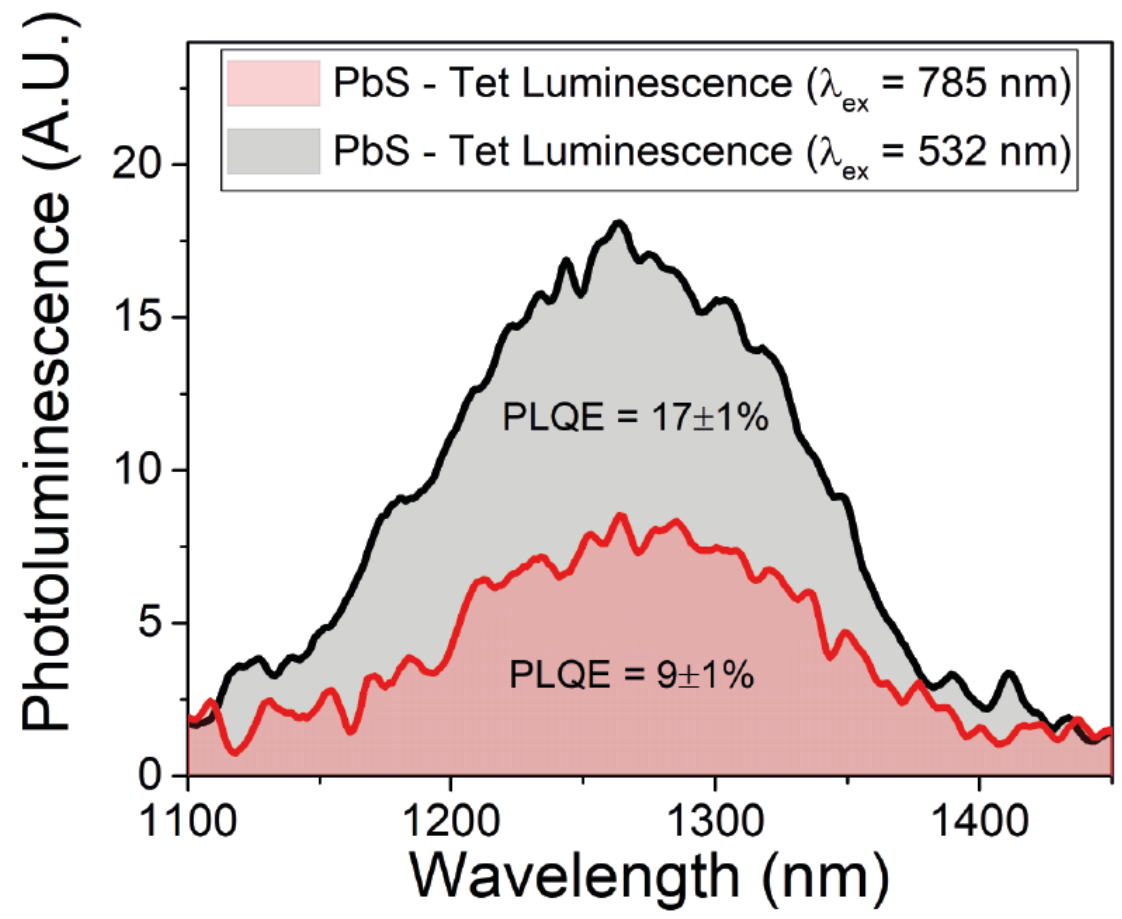

Figure 3: PLQE and PL spectrum of PbS-Tet excited at $532 \mathrm{~nm}$ and $785 \mathrm{~nm}$. At $785 \mathrm{~nm}$ photons are absorbed only in the PbS and at $532 \mathrm{~nm}$ photons are absorbed mainly in the TetCAL ligands. We note that the PLQE measurements presented at absolute values and take into account any changes in absorption and scattering between the different excitation wavelengths.

Figure 4a shows the transient photoluminescence (PL) dynamics of the PbS in the PbS-tet system, measured via time-correlated single-photon counting (TCSPC). When excited at $650 \mathrm{~nm}$, where only the PbS absorbs, the PL decays with a lifetime of $1.4 \mu \mathrm{s}$. When excited at $530 \mathrm{~nm}$ where the TetCAL ligand strongly absorbs the PL decays with a lifetime of $1.8 \mu \mathrm{s}$. The measured decays are independent of emission wavelength. We ascribe the increased QD PL lifetime following ligand excitation to the transfer of long-lived triplets from the TetCAL to the $\mathrm{PbS}$ nanocrystal. This agrees with similar results reported in $\mathrm{PbS} Q \mathrm{QD} /$ tetracene bilayers $^{10}$. No such variation of lifetime with excitation wavelength was seen in PbS-OA QDs (see SI). The inset in figure 4a plots the difference in the PL kinetics at early times when the PbS-tet is excited at $530 \mathrm{~nm}$ vs $650 \mathrm{~nm}$. As can be seen, a rise in PL is detected (within the differential PL signal) with a time constant of 60ns, indicative of energy transfer into the $\mathrm{PbS}$ on this time scale. This timescale is comparable to previous work reported in $\mathrm{PbS} Q \mathrm{QD} /$ tetracene bilayers $^{10}$. 
Figure $4 \mathrm{~b}$ shows transient absorption (TA) spectra of the PbS-tet QDs following photoexcitation at $532 \mathrm{~nm}$. Full details of TA measurement on concentrated solutions of TetCAL ligands and PbS-OA QDs are presented in the $\mathrm{SI}$; these measurements are used to assign the spectral features in PbS-tet presented here. At early times (1 ps) following photoexcitation the TA spectrum is consistent with the singlet state of TetCAL superimposed on a broad signal from the PbS QDs (see SI). A ground-state bleach (GSB) feature of TetCAL can be seen around $550 \mathrm{~nm}$, stimulated emission (SE) at $600 \mathrm{~nm}$ and photoinduced absorption (PIA) from the singlet exciton at $650 \mathrm{~nm}$. This entire spectrum is pulled below zero by the broad signal from the PbS. By 5 ns, the SE and PIA associated with the singlet are replaced with PIA from the TT state. By 50 ns the TT states decay to form free triplets and excitations of the PbS, which give a strong PIA at $620 \mathrm{~nm}$, suggesting the transfer of one of the triplets into the PbS, while the other remains on the TetCAL ligand. At present, it is not possible to tell if the TT state breaks up prior to triplet injection, or if one of the triplets is injected directly from the TT state. At later times, $5 \mu \mathrm{s}$, we detect signatures consistent with free triplets on the TetCAL ligand, but the PIA associated with the excitons on the PbS at $620 \mathrm{~nm}$ is lost. Based on these spectra we suggest that the photoexcitation in the QDs recombines before the transfer of the remaining triplets from the TetCAL ligand. These remaining triplets have a lifetime of $14 \mu \mathrm{s}$, much shorter than the $70 \mu$ s lifetime of triplets formed via fission in concentrated solutions of TetCAL ligands. This shows that the triplets on the TetCAL ligands attached to the QDs are efficiently quenched via transfer to the QDs. We note that if the both triplets were transfer to the QD at the same time we would expect to see a reduction in PLQE as biexction populations have been shown to reduce PLQE in PbS and other QDs due to Auger processes ${ }^{23,24}$.
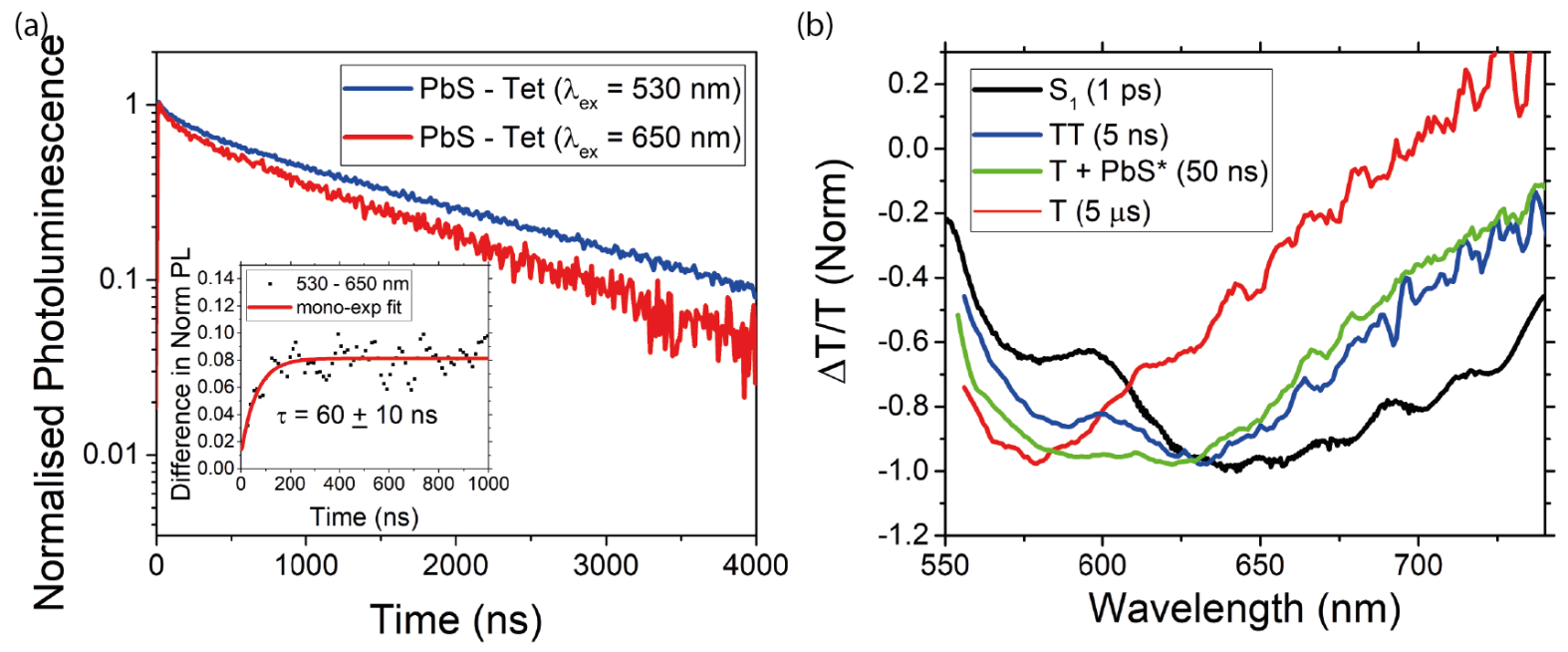

Figure 4: (a) Transient photoluminescence of PbS -Tet under $530 \mathrm{~nm}$ and $650 \mathrm{~nm}$ excitation measured via TCSPC at an emission wavelength of $1250 \mathrm{~nm}$. The PL lifetime is extended to $1.8 \mu \mathrm{s}$ from $1.4 \mu \mathrm{s}$ when excited at $530 \mathrm{~nm}$ vs $650 \mathrm{~nm}$. The inset shows the difference of the two $\mathrm{PL}$ traces, giving a rise time of 60ns, which is consistent with the triplet transfer time into QDs observed in previous studies (b) TA spectra of PbS - Tet excited at 532nm. As described in the main text, the 1 ps spectrum is consistent with the singlet exciton of TetCAL superimposed on 
a broad signal from the PbS. At 5 ns a spectrum consistent with TT states is observed, at 50 ns the spectrum is consistent with a superposition of triplet excitons on TetCAL and excitations on the $\mathrm{PbS}$, and at $5 \mu \mathrm{s}$ the spectrum is consistent with triplet excitons on TetCAL.

Overall, the TA data are consistent with the formation of TT states from the initially photogenerated singlet state on the TetCAL ligands. Triplets are then injected into the QDs, where they form an excited state in the QD and subsequently recombine. Following this the remaining triplets are injected into the $Q D$, where they can then recombine. More detailed future studies are called for to clarify the exact mechanism and sequence of the triplet injection process.

In conclusion, we have demonstrated that using singlet fission molecules as ligands for QDs allows for the doubling of QD PLQE when the photons are absorbed by the ligand, as compared to when directly absorbed in the QD. This doubling of the PLQE of the QDs, shows that both the singlet fission process in the ligands as well as the subsequent injection of the triplets into the QDs must occur with near unity efficiency. This is a very surprising result for the reasons described in detail above. Our results raise a number of questions which will need to be probed by future studies, for instance, (i) the mechanism of fission in the ligands attached to the QDs, (ii) if dynamic structural rearrangements of the ligand shell aid in the formation and breakup of the TT states, (iii) if triplets can be injected directly from the TT states into the QDs, and (iv) if the injection of one triplet blocks the injection of another triplet from the ligands thus preventing the formation of biexcitons and enabling efficient sequential injection.

Turing back to equation 1, which describes the overall quantum efficiency of a photon multiplication process based on singlet fission, we find that the approach demonstrated here collectively maximises the efficiency of each step of the process except $\eta_{\mathrm{PL}}$, the efficiency of light emission from the QDs. However, it should be possible to independently optimise this step, for example via the use of post-ligand-exchange surface passivation, without compromising the other steps. Thus, our approach holds great promise for achieving a photon multiplication yield close to the theoretical maximum of $200 \%$ and provides a clear path towards the harnessing of singlet fission to enhance the efficiency of inorganic photovoltaics and ultimately to overcome the Shockley-Queisser limit.

\section{Experimental Methods}

Materials: All chemicals were purchased from Sigma-Aldrich and used as delivered. The TIPSTetracene-Carboxylic acid (TetCA) was synthesized as described previously ${ }^{25}$.

PbS nanocrystal synthesis: The synthesis of PbS QDs was carried out following modified versions of previously reported methods ${ }^{26}$. Briefly, $\mathrm{PbO}(0.62 \mathrm{~g}, 2.8 \mathrm{mmol})$, oleic acid $(2.5 \mathrm{ml}, 7.8 \mathrm{mmol}, 1.81 \mathrm{~g}$ (acceptor) or $1.5 \mathrm{ml}, 4.7 \mathrm{mmol}, 1.3 \mathrm{~g}$ (donor) and octadecene $(25.0 \mathrm{ml}, 76.3 \mathrm{mmol}, 19.5 \mathrm{~g})$ were combined in a three-neck flask and degassed at $110^{\circ} \mathrm{C}$ under vacuum $\left(10^{-2}\right.$ mbar or better) for $2 \mathrm{~h}$. Subsequently, the reaction flask was flushed 
with nitrogen and heated to $115^{\circ} \mathrm{C}$. A solution of bis(trimethylsilyl)sulphide (286 $\mu \mathrm{l}, 1.4$ $\mathrm{mmol})$, diphenylphosphine $(144 \mu \mathrm{l}, 0.83 \mathrm{mmol})$ in octadecene $(13.9 \mathrm{ml}, 42.4 \mathrm{mmol}, 10.8 \mathrm{~g})$ was rapidly injected into the lead precursor solution. The bandgap of the PbS QDs was tuned by adjusting the oleic acid concertation as above. The reaction was quenched by placing the reaction flask in an ice-water bath. The PbS QDs were isolated from the reaction mixture by flocculating to turbidity using a 1-buthanol/ethanol/hexane solvent system. The purified QDs were then redispersed in octane at a concentration of $\sim 100 \mathrm{mg} \mathrm{ml}^{-1}$ and stored under argon.

Ligand exchange: The ligand exchange was performed using a modified literature procedure ${ }^{25}$. Previously synthesized PbS-OA was used, in the presence of TetCAL in toluene. TetCAL (variable amounts) was added to a $20 \mathrm{mg} / \mathrm{ml}$ solution of $\mathrm{PbS}-\mathrm{OA}$ in $1 \mathrm{ml}$ toluene and the resulting solution was maintained in the dark for 16 hours. Then, acetone $(4 \mathrm{~mL})$ was added to precipitate the PbS-tet nanocrystals; the mixture was centrifuged at $4000 \mathrm{rpm}$ for 5 minutes, the supernatant was removed, and the residue was dispersed in toluene $(1 \mathrm{~mL})$. The PbS-tet nanocrystals were purified using a minimum of 6 repeated dispersion/precipitation/centrifugation cycles. Finally, PbS-OA/TPn nanocrystals were dispersed in toluene $(1 \mathrm{~mL})$, and stored under nitrogen in the dark at $-20^{\circ} \mathrm{C}$.

Continuous wave measurements: Absorption spectra of solutions were measured on nanocrystals samples dispersed in toluene at a concentration of ca. $1 \mathrm{mg} \mathrm{mL}^{-1}$ in a $0.1 \mathrm{~cm} \times 1$ $\mathrm{cm}$ cuvette using a Shimudzu UV600 spectrometer with attached integrating sphere. Photoluminescence excitation spectra were measured on an Edinburgh Instruments FLS90 fluorimeter. Solution samples were measured in a $1 \mathrm{~cm} \times 0.3 \mathrm{~cm}$ cuvette excited in the $1 \mathrm{~cm}$ direction and imaged in the $0.3 \mathrm{~cm}$ direction.

PLQE measurements: Nanocrystal solutions were placed in an integrating sphere and were photo-excited using $532 \mathrm{~nm}$ and $785 \mathrm{~nm}$ continuous-wave lasers. The laser and the emission signals were measured and quantified using a calibrated Andor iDus DU490A InGaAs detector for the determination of PL quantum efficiency. PLQE was calculated using the method of de Mello et al. ${ }^{27}$.

Briefly, PLQE was calculated as $\frac{\text { number of photons emitted }}{\text { number of photons absorbed }}$. To calculate these numbers three measurements were performed in an integrating sphere and the total light intensity received at the spectrometer was measured. a) laser excitation with no sample, b) laser excitation and sample emission with direct illumination of sample and c) laser excitation and sample emission with indirect illumination of sample. Integrating the excitation and emission signals allows the follow calculations.

Number of photons absorbed $=L_{a} A$, where $L_{a}$ is the excitation intensity in experiment a. and $A=\left(1-\frac{L_{c}}{L_{b}}\right)$, where $L_{b}$ and $L_{c}$ are the excitation intensities in experiment $b$ and $c$ respectively.

Number of photons emitted $=P_{c}-(1-A) P_{b}$, where $P_{b}$ and $P_{c}$ are the emission intensities in experiment $b$ and $c$. 
Thus PLQE is calculated as $P L Q E=\frac{P_{c}-(1-A) P_{b}}{L_{a} A}$.

This method accounts for all photons absorbed by direct excitation, indirect excitation via scattering in the integration sphere and sample emission. Full details can be found in the de Mello et al. publication ${ }^{25}$."

We note that the fluence used for these measurements was kept low to avoid two-photon absorption. The error in the calculated PLQE values is $\pm 1 \%$ (absolute).

Transmission electron microscopy: TEM samples were prepared by drop casting a ca. $5 \mathrm{mg}$ $\mathrm{mL}^{-1}$ nanocrystal solution in toluene on a TEM Grid (200 Mesh Cu, Agar Scientific) in an argonfilled glove box and imaged employing a FEI Tecnai F20 microscope operated at $200 \mathrm{kV}$. Nanocrystal size distributions were measured using the software package ImageJ (https://imagej.nih.gov/ij/). A contrast threshold was chosen which solely included the nanocrystal particles and not the image background. Using the analyze particle function, which counts areas of contrast above the threshold, the size distribution was measured. Particle size was limited to a designated maximum which excluded overlaid nanocrystals. This was done for 5 of different TEM images.

Transient Absorption: In this technique a pump pulse generates photoexcitations within the solutions, which are then probed at later times using a broadband probe pulse. Transient absorption spectra were recorded over short (50 fs - $2 \mathrm{~ns}$ ) and long ( $1 \mathrm{~ns}-500 \mu \mathrm{s}$ ) time delays with a probe pulse covering from $550-750 \mathrm{~nm}$. For short-time (ps-TA) measurements a portion of the output of a Ti:Sapphire amplifier system (Spectra-Physics Solstice) operating at $1 \mathrm{kHz}$, was used to pump a home built non-collinear optical parametric amplifier (NOPA) to generate the pump pulse at $532 \mathrm{~nm}$ (FWHM $50 \mathrm{~nm},<100 \mathrm{fs}$ ). In both short and long-time measurements another portion of the amplifier output was used to pump a home-built noncollinear optical parametric amplifier, to generate the probe pulse. The probe beam was then split to generate a reference beam so that laser fluctuations could be normalized. For shorttime measurements the probe is delayed using a mechanical delay-stage (Newport). For longtime (ns-TA) measurements a separate frequency-doubled Q-switched Nd:YVO4 laser (AOTYVO-25QSPX, Advanced Optical Technologies) is used to generate the pump. This laser produces pulses with a temporal breadth below $1 \mathrm{~ns}$ at $530 \mathrm{~nm}$ and has an electronically controlled delay. The pump and probe beams are overlapped on the sample adjacent to a reference probe beam. This reference is used to account for any shot-to-shot variation in transmission. The sample is held in a $1 \mathrm{~mm}$ quartz cuvette, mounted into a holder. The beams are focused into an imaging spectrometer (Andor, Shamrock SR 303i) and detected using a pair of linear image sensors (Hamamatsu, G11608) driven and read out at the full laser repetition rate by a custom-built board from Stresing Entwicklungsburo. Initial measurements were recorded at a range of laser fluences $\left(30-400 \mu / \mathrm{cm}^{2}\right)$ on both the ps and ns transient absorption set-ups. The TA spectra presented in this work were recorded at $150 \mu \mathrm{J} / \mathrm{cm}^{2}$. In all measurements every second pump shot is omitted, either electronically for long-time measurements or using a mechanical chopper for short-time measurements. The fractional differential transmission $(\Delta T / T)$ of the probe is calculated for each data point once 1000 shots have been collected. 
TCSPC: Transient PL measurements with TCSPC. The sample was excited with a pulsed supercontinuum laser (Fianum Whitelase SC-400-4, 6 ps pulse lengths) at $0.2 \mathrm{MHz}$ repetition rate. The pump wavelength was selected to $530 \mathrm{~nm}$ or $650 \mathrm{~nm}$ (full-width at half-maximum $10 \mathrm{~nm}$ ) with dielectric filters (Thorlabs). Pump scatter light from the laser excitation within the photoluminescence path to the detector was filtered-out using an absorptive long-pass filter with a $1000 \mathrm{~nm}$ edge (Thorlabs). The photoluminescence was focused and detected by a single-photon avalanche photodiode based on InGaAs/InP (MPD-InGaAs-SPAD).

\section{Acknowlegdments:}

N. J. L. K. D. acknowledges the Ernest Oppenheimer fund for an Oppenheimer Early Career Research Fellowship. J. R. A. acknowledges Cambridge Commonwealth European and International Trust for financial support. J. X. acknowledges EPSRC CDT in Nanoscience and Nanotechnology for financial support. The authors thank the Winton Programme for the Physics of Sustainability and the Engineering and Physical Sciences Research Council for funding. The data underlying this publication are available at https://doi.org/10.17863/CAM.20890.

\section{Supporting Information}

The Supporting Information is available free of charge on the ACS Publications website at DOI:

The supporting information contains the following: Absorption spectra of $\mathrm{PbS}-\mathrm{Tet}, \mathrm{PbS}-$ $\mathrm{OA}$ and TetCal in toluene; TEM images and size distribution of PbS - OA QDs and PbS - Tet QDs; Extinction coefficient spectra of TetCAL; Photoluminescence spectra of PbS QDs with attached TetCAL ligands; PLQE and PL spectrum of PbS-OA excited with $532 \mathrm{~nm}$ and $785 \mathrm{~nm}$ illumination; Life times extracted by decay associated spectra and TCSPS fits. Further transient spectroscopy data including absorption and luminesces of $\mathrm{PbS}-\mathrm{OA}, \mathrm{PbS}-\mathrm{Tet}$ and TetCAL sotuions.

\section{References}

(1) Stern, H. L.; Musser, A. J.; Gelinas, S.; Parkinson, P.; Herz, L. M.; Bruzek, M. J.; Anthony, J.; Friend, R. H.; Walker, B. J. Identification of a Triplet Pair Intermediate in Singlet Exciton Fission in Solution. Proc. Natl. Acad. Sci. 2015, 112, 7656-7661.

(2) Smith, M. B.; Michl, J. Recent Advances in Singlet Fission. Annu. Rev. Phys. Chem. 2013, 64, 361-386.

(3) Rao, A.; Friend, R. H. Harnessing Singlet Exciton Fission to Break the Shockley-Queisser Limit. Nat. Rev. Mater. 2017, 2, 1-12.

(4) Shockley, W.; Queisser, H. J. Detailed Balance Limit of Efficiency of P-N Junction Solar Cells. J. Appl. Phys. 1961, 32, 510.

(5) Green, M. A.; Emery, K.; Hishikawa, Y.; Warta, W.; Dunopl, E. D. Solar Cell Efficiency Tables (Version 44). Prog. Photovolt. Res. Appl. 2014, 22, 701-710.

(6) Lee, J.; Jadhav, P.; Reusswig, P. D.; Yost, S. R.; Thompson, N. J.; Congreve, D. N.; Hontz, E.; Van 
Voorhis, T.; Baldo, M. A. Singlet Exciton Fission Photovoltaics. Acc. Chem. Res. 2013, 46, $1300-1311$.

(7) Nozik, A. J. Nanoscience and Nanostructures for Photovoltaics and Solar Fuels. Nano Lett. 2010, 10, 2735-2741.

(8) Ehrler, B.; Wilson, M. W. B.; Rao, A.; Friend, R. H.; Greenham, N. C. Singlet Exciton FissionSensitized Infrared Quantum Dot Solar Cells. Nano Lett. 2012, 12, 1053-1057.

(9) Pazos-Outón, L. M.; Lee, J. M.; Futscher, M. H.; Kirch, A.; Tabachnyk, M.; Friend, R. H.; Ehrler, B. A Silicon-Singlet Fission Tandem Solar Cell Exceeding 100\% External Quantum Efficiency with High Spectral Stability. ACS Energy Lett. 2017, 2, 476-480.

(10) Thompson, N. J.; Wilson, M. W. B.; Congreve, D. N.; Brown, P. R.; Scherer, J. M.; Bischof, T. S.; Wu, M.; Geva, N.; Welborn, M.; Voorhis, T. Van; et al. Energy Harvesting of Non-Emissive Triplet Excitons in Tetracene by Emissive PbS Nanocrystals. Nat. Mater. 2014, 13, 1039-1043.

(11) Tabachnyk, M.; Ehrler, B.; Gélinas, S.; Böhm, M. L.; Walker, B. J.; Musselman, K. P.; Greenham, N. C.; Friend, R. H.; Rao, A. Resonant Energy Transfer of Triplet Excitons from Pentacene to PbSe Nanocrystals. Nat. Mater. 2014, 13, 1033-1038.

(12) Yost, S. R.; Lee, J.; Wilson, M. W. B.; Wu, T.; McMahon, D. P.; Parkhurst, R. R.; Thompson, N. J.; Congreve, D. N.; Rao, A.; Johnson, K.; et al. A Transferable Model for Singlet-Fission Kinetics. Nat. Chem. 2014, 6, 492-497.

(13) Tabachnyk, M.; Ehrler, B.; Gélinas, S.; Böhm, M. L.; Walker, B. J.; Musselman, K. P.; Greenham, N. C.; Friend, R. H.; Rao, A. Resonant Energy Transfer of Triplet Excitons from Pentacene to PbSe Nanocrystals. Nat. Mater. 2014, 13, 1033-1038.

(14) Nienhaus, L.; Wu, M.; Geva, N.; Shepherd, J. J.; Wilson, M. W. B.; Bulović, V.; Van Voorhis, T.; Baldo, M. A.; Bawendi, M. G. Speed Limit for Triplet-Exciton Transfer in Solid-State PbS Nanocrystal-Sensitized Photon Upconversion. ACS Nano 2017, 11, 7848-7857.

(15) Huang, Z.; Li, X.; Mahboub, M.; Hanson, K. M.; Nichols, V. M.; Le, H.; Tang, M. L.; Bardeen, C. J. Hybrid Molecule-Nanocrystal Photon Upconversion Across the Visible and Near-Infrared. Nano Lett. 2015, 15, 5552-5557.

(16) Huang, Z.; Tang, M. L. Designing Transmitter Ligands That Mediate Energy Transfer between Semiconductor Nanocrystals and Molecules. J. Am. Chem. Soc. 2017, 139, 9412-9418.

(17) Mongin, C.; Garakyaraghi, S.; Razgoniaeva, N.; Zamkov, M.; Castellano, F. N. Direct Observation of Triplet Energy Transfer from Semiconductor Nanocrystals. Science 2016, 351, 369-372.

(18) Mahboub, M.; Huang, Z.; Tang, M. L. Efficient Infrared-to-Visible Upconversion with Subsolar Irradiance. Nano Lett. 2016, 16, 7169-7175.

(19) Huang, Z.; Xu, Z.; Mahboub, M.; Li, X.; Taylor, J.; Harman, H.; Lian, T.; Tang, M. PbS/CdS Core/shell QDs Suppress Charge Transfer and Enhance Triplet Transfer. Angew. Chemie Int. Ed. 2017, 56, 16583-16587.

(20) Johnson, J. C.; Nozik, A. J.; Michl, J. The Role of Chromophore Coupling in Singlet Fission. Acc. Chem. Res. 2013, 46, 1290-1299.

(21) Stern, H. L.; Cheminal, A.; Yost, S. R.; Broch, K.; Bayliss, S. L.; Chen, K.; Tabachnyk, M.; Thorley, K.; Greenham, N.; Hodgkiss, J. M.; et al. Vibronically Coherent Ultrafast Triplet-Pair Formation and Subsequent Thermally Activated Dissociation Control Efficient Endothermic Singlet Fission. Nat. Chem. 2017, 9, 1205-1212. 
(22) Moreels, I.; Justo, Y.; De Geyter, B.; Haustraete, K.; Martins, J. C.; Hens, Z. Size-Tunable, Bright, and Stable PbS Quantum Dots: A Surface Chemistry Study. ACS Nano 2011, 5, 20042012.

(23) Ellingson, R. J.; Beard, M. C.; Johnson, J. C.; Yu, P.; Micic, O. I.; Nozik, A. J.; Shabaev, A.; Efros, A. L. Highly Efficient Multiple Exciton Generation in Colloidal PbSe and PbS Quantum Dots. Nano Lett. 2005, 5, 865-871.

(24) Klimov, V. I.; Mikhailovsky, A. A.; McBranch, D. W.; Leatherdale, C. A.; Bawendi, M. G. Quantization of Multiple Auger Rates in Semiconductor Quantum Dots. Science 2000, 287, 1011-1013.

(25) Garakyaraghi, S.; Mongin, C.; Granger, D. B.; Anthony, J. E.; Castellano, F. N. Delayed Molecular Triplet Generation from Energized Lead Sulfide Quantum Dots. J. Phys. Chem. Lett. 2017, 8, 1458-1463.

(26) Hines, M. A.; Scholes, G. D. Colloidal PbS Nanocrystals with Size-Tunable Near-Infrared Emission: Observation of Post-Synthesis Self-Narrowing of the Particle Size Distribution. Adv. Mater. 2003, 15, 1844-1849.

(27) Mello, J. de; Wittmann, H.; Friend, R. An Improved Experimental Determination of External Photoluminescence Quantum Efficiency. Adv. Mater. 1997, 9, 230-232. 


\section{Supporting Information}

Singlet Fission and Triplet Transfer to PbS Quantum Dots in TIPS-Tetracene Carboxylic Acid Ligands

Nathaniel J. L. K. Davis ${ }^{a}$, Jesse R. Allardice ${ }^{a}$, James Xiao ${ }^{a}$, Anthony J. Petty II ${ }^{b}$, Neil C. Greenham ${ }^{\mathrm{a}}$, John E. Anthony ${ }^{\mathrm{b}}$ and Akshay Rao ${ }^{\mathrm{a} *}$.

\section{Contacts}

${ }^{a}$ Cavendish Laboratory, University of Cambridge, J.J. Thomson Avenue, Cambridge, CB3 OHE, UK

${ }^{b}$ Department of Chemistry, University of Kentucky ,161 Jacobs Science Building, Lexington KY 405060174

*ar525@cam.ac.uk 


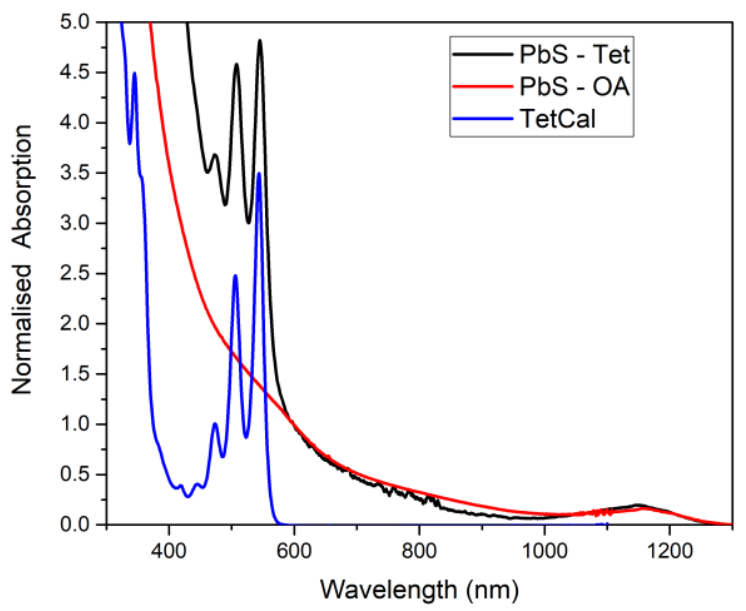

Figure S1: Absorption spectra of $\mathrm{PbS}-\mathrm{Tet}, \mathrm{PbS}-\mathrm{OA}$ and TetCal in toluene.
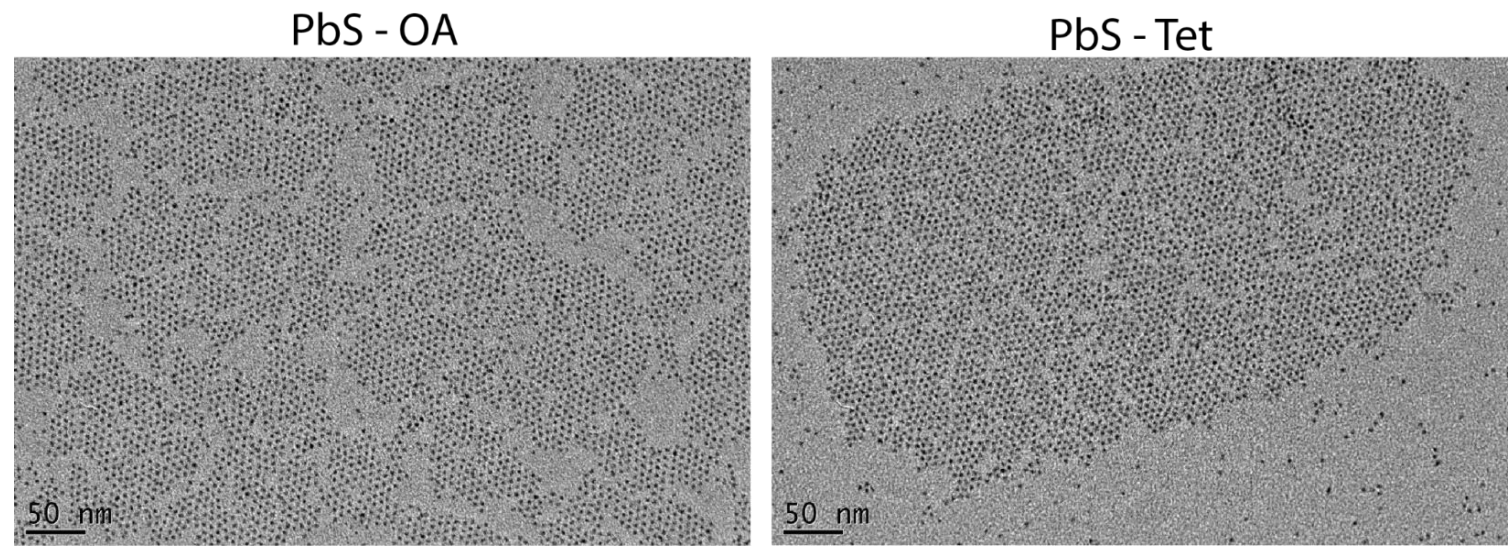

Figure S2: TEM images of PbS - OA QDs and PbS - Tet QDs. 


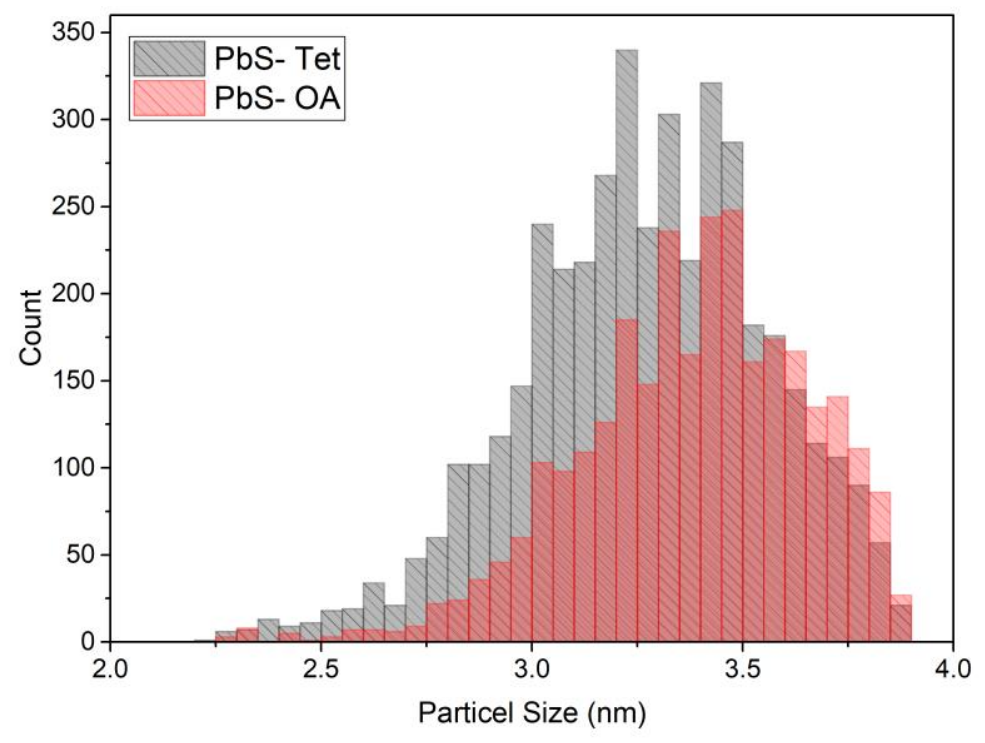

Figure S3: Size distribution from TEM PbS - OA QDs and PbS - Tet QDs. The slight difference in particles size is most likely the result of the difference in ligand lengths of oleic acid and TetCal. 


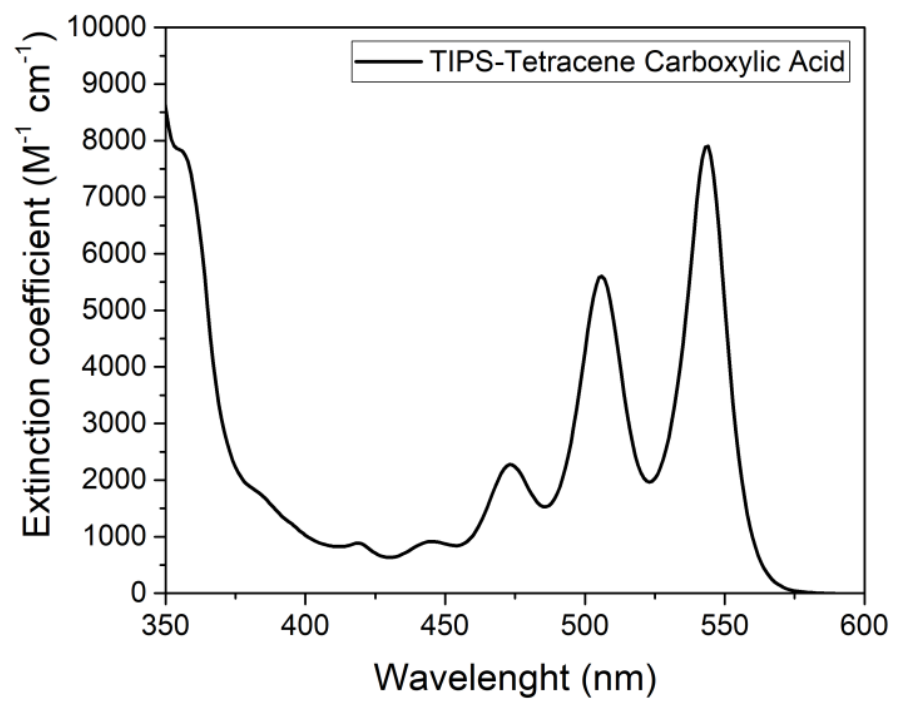

Figure S4: Extinction coefficient of TetCAL, measured from a dilute solution of TetCAL in Toluene.

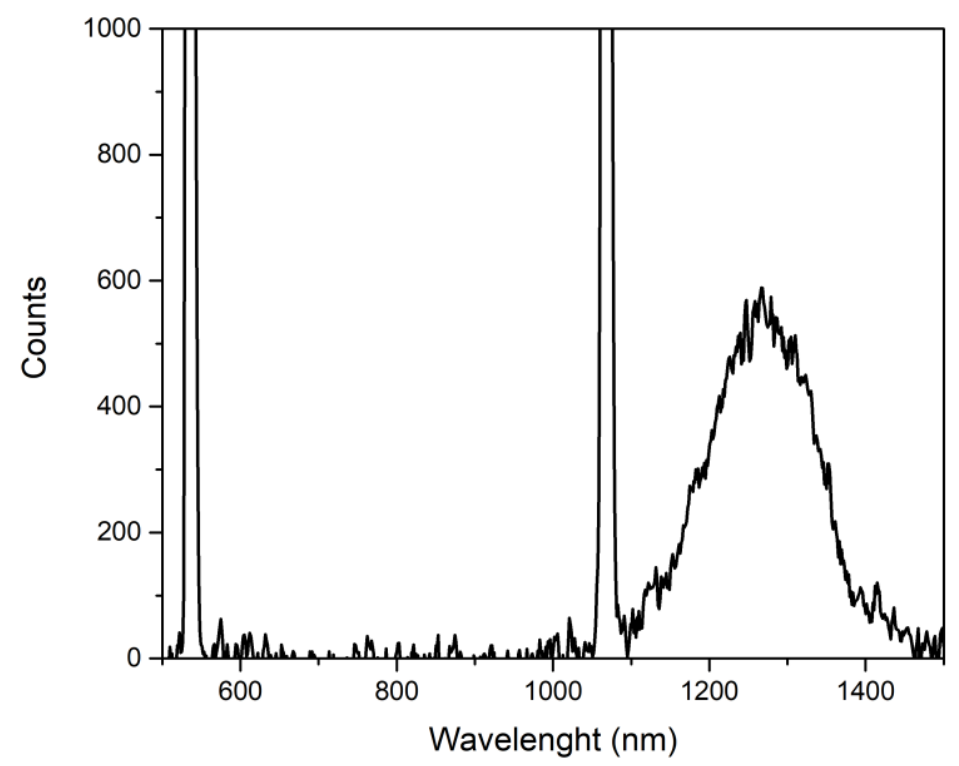

Figure S5: Photoluminescence spectra of PbS QDs with attached TetCAL ligands under $532 \mathrm{~nm}$ excitation. The spectra demonstrates that the PL of the TetCAL ligand is completely quenched upon attachment to the PbS. The sharp peaks at $532 \mathrm{~nm}$ and $1064 \mathrm{~nm}$ are the excitation laser and its second harmonic from the grating of the spectrometer. 


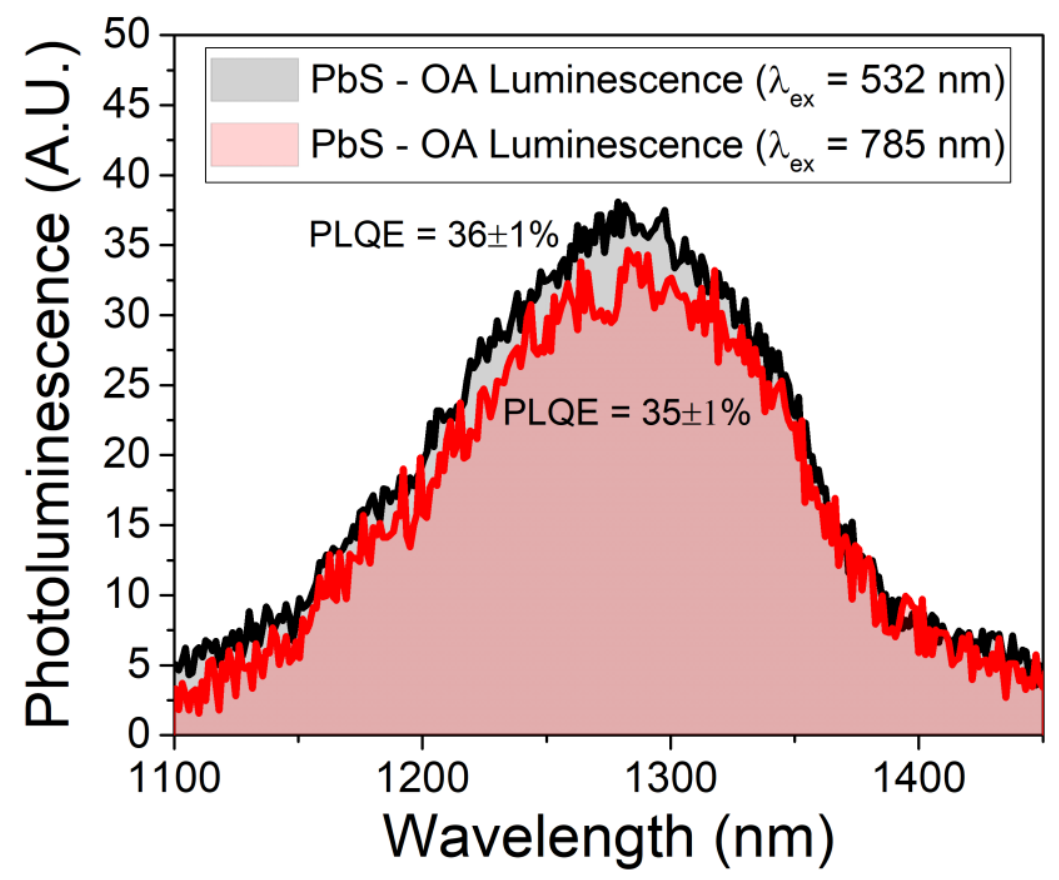

Figure S6: PLQE and PL spectrum of PbS-OA excited with $532 \mathrm{~nm}$ and $785 \mathrm{~nm}$ illumination at $20 \mu \mathrm{W}$. For these QDs not increase in PLQE is observed under the two excitation wavelengths, in contrast to the the QDs with TetCAl ligands (Figure 3).

Table S1: Life times extracted by decay associated spectra and TCSPS fits

\begin{tabular}{|l|l|l|l|l|l|}
\hline \multicolumn{3}{|c|}{ DAS on TA data } & \multicolumn{3}{c|}{ TCSPC } \\
\hline TetCAL & Lifetime (ns) & Error (ns) & PbS & Lifetime (ns) & Error (ns) \\
\hline TT & 15.05 & 0.07 & @ $650 \mathrm{~nm}$ & 2022.45 & 7.20 \\
\hline Triplet & 69762.52 & 1201.63 & @ 530 nm & 1930.48672 & 13.60 \\
\hline PbS & Lifetime (ns) & Error (ns) & PbS - Tet & Lifetime (ns) & Error (ns) \\
\hline Exciton & 2170.00 & 20.00 & @ 650 nm & 1396.57 & 27.96 \\
\hline PbS- Tet & Lifetime (ns) & Error (ns) & @ 530 nm & 1830.93 & 25.51 \\
\hline Singlet & 3.53 & 0.30 & & & \\
\hline TT & 44.28 & 2.01 & & & \\
\hline Triplet & 1778.45 & 58.57 & & & \\
\hline
\end{tabular}




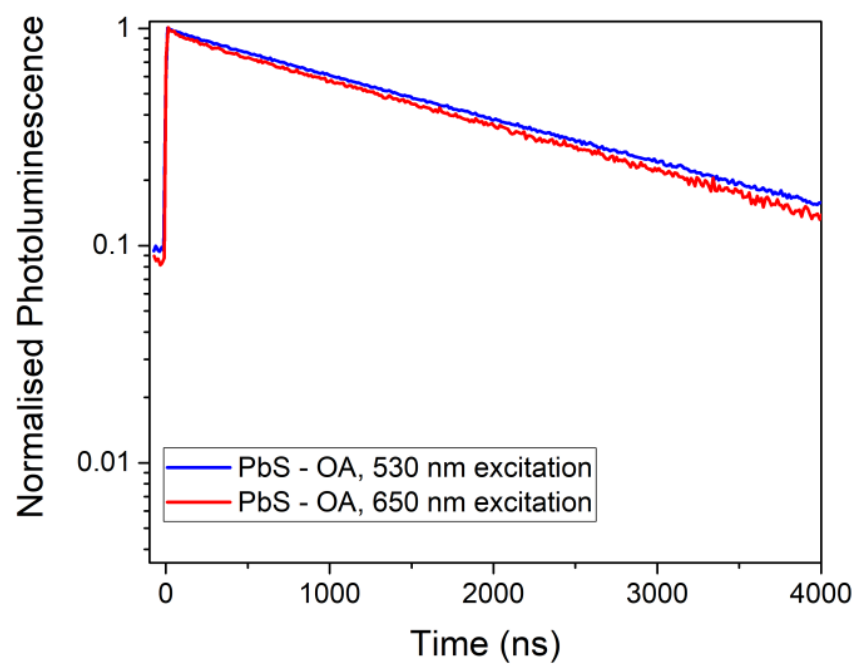

Figure S7: Transient photoluminescence of $\mathrm{PbS}-\mathrm{OA}$ under $530 \mathrm{~nm}$ and $650 \mathrm{~nm}$ excitation $\left(6 \mu \mathrm{W}, 24 \mathrm{~nJ} / \mathrm{cm}^{2}\right)$, measured via TCSPC.

We observe no dependence of the transient PL of PbS-OA on the wavelength of excitation. Figure S7 shows the near equivalence of the transient PL dynamics of PbS-OA QDs under $6 \mu \mathrm{W}$ of $530 \mathrm{~nm}$ and $650 \mathrm{~nm}$ pulsed excitation. This is in contrast to PbS-Tet where we see a difference in lifetimes under exciton with $530 \mathrm{~nm}$ and $650 \mathrm{~nm}$ excitation (Figure 4 (a)).

To ensure that any observed differences in decay dynamics are due to transfer and not multi-exciton effects, such as exciton anihilation, TCSPC measurements were performed at multiple excitation fluences. Using $650 \mathrm{~nm}$ excitation with of laser fluences between $0.4-24 \mathrm{~nJ} / \mathrm{cm}^{2}(100-6000 \mathrm{nW})$, we see no change in the PL decay dynamics of the PbS-OA QDs (figure S8 (a)). Additionally using $530 \mathrm{~nm}$ excitation with laser fluences between $6-24 \mathrm{~nJ} / \mathrm{cm}^{2}(1600-6000 \mathrm{nW})$, we see no change in the PL decay dynamics of the PbS-Tet QDs (Figure $\mathrm{S} 8(\mathrm{~b})$ ). Therefore, the measurements are in the linear excitation regime and $24 \mathrm{~nJ} / \mathrm{cm}^{2}(6 \mu \mathrm{W})$ of $530 \mathrm{~nm}$ or $650 \mathrm{~nm}$ laser excitation will result in dynamics which are not influenced by multi-exciton effects. 
(a) $\mathrm{PbS}-\mathrm{OA}$

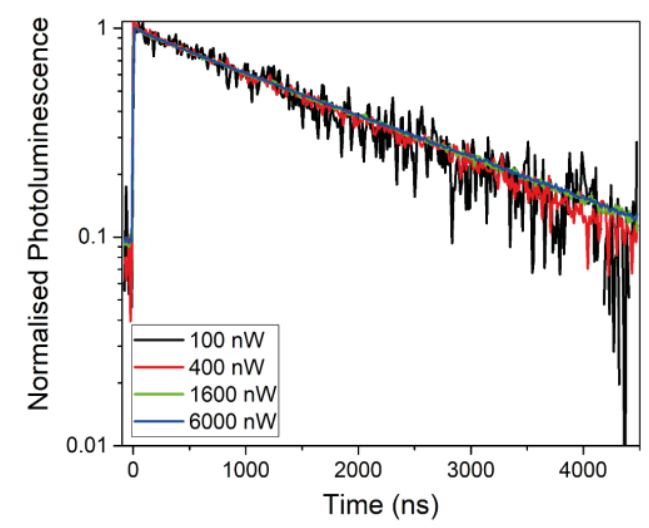

(b) PbS - Tet

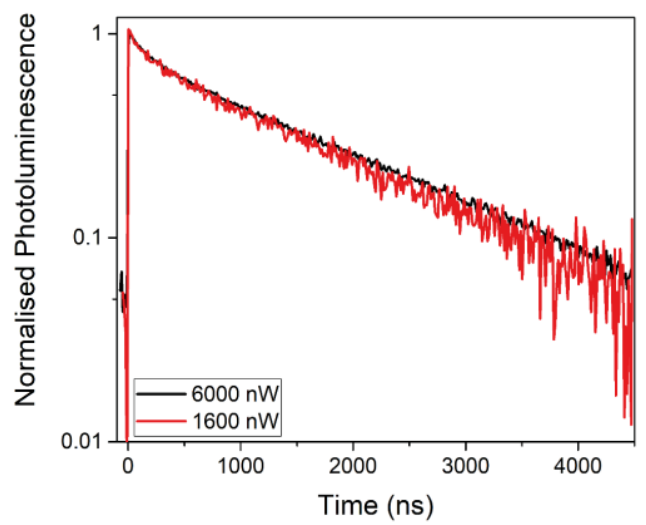

Figure S8: Fluence dependence of transient photoluminescence in $\mathrm{PbS}-\mathrm{OA}(\mathrm{a})$ and $\mathrm{PbS}-\mathrm{Tet}$ (b) under $650 \mathrm{~nm}$ excitation.

Similar to previously reported singlet fission in concentrated TIPS-Tetracene solutions (reference 1 of main text), we find that in concentrated solutions $(0.5 \mathrm{M})$ of TetCAL (free ligands in solution with no $Q D s)$, triplets form via diffusion and collisions. These triplets decay with a lifetime of $70 \mu \mathrm{s}$.

A clear conversion between $T T$ states formed via collisions at early times and free triplets formed via breakup of TT states is detected consentient with previous work on TIPS-Tetracene solutions. Decay associated spectra (DAS) analysis confirms the presence of 2 species.

(a)

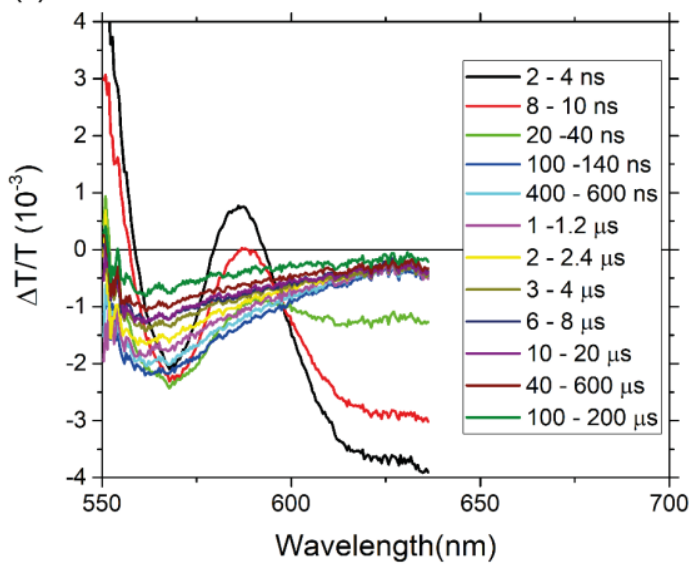

(b)

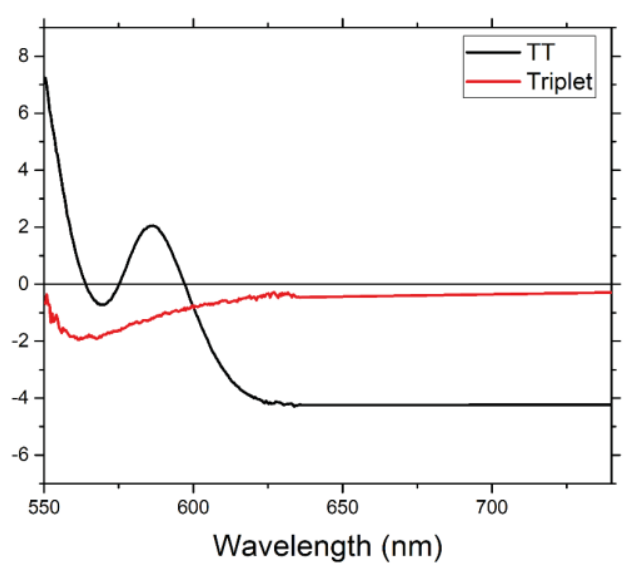

Figure S9: (a) Time transient absorption spectra of TetCAL only solutions (free ligands in solution) using $532 \mathrm{~nm}$ excitation $\left(400 \mu \mathrm{J} / \mathrm{cm}^{2}\right)$. (b) DAS of spectra showing the 2 compoents.

After photoexcitation untreated PbS QDs show a broad photo-induced absorption (PIA) feature peaking at $620 \mathrm{~nm}$ which decays with a time constant of $2170 \mathrm{~ns}$ (Figure 4 (a) and SI Figure 10 (a)), in agreement with the transient PL measurements.

DAS was performed on the ns transient absorption data. The DAS calculated for the PbS excitation in shown in figure S10 (b). The DAS shows that the spectrum consists of single component consistent with the know spectra of PbS. All DAS lifetimes and exponential fits to the TCSPC data can be found in SI Table 1. 

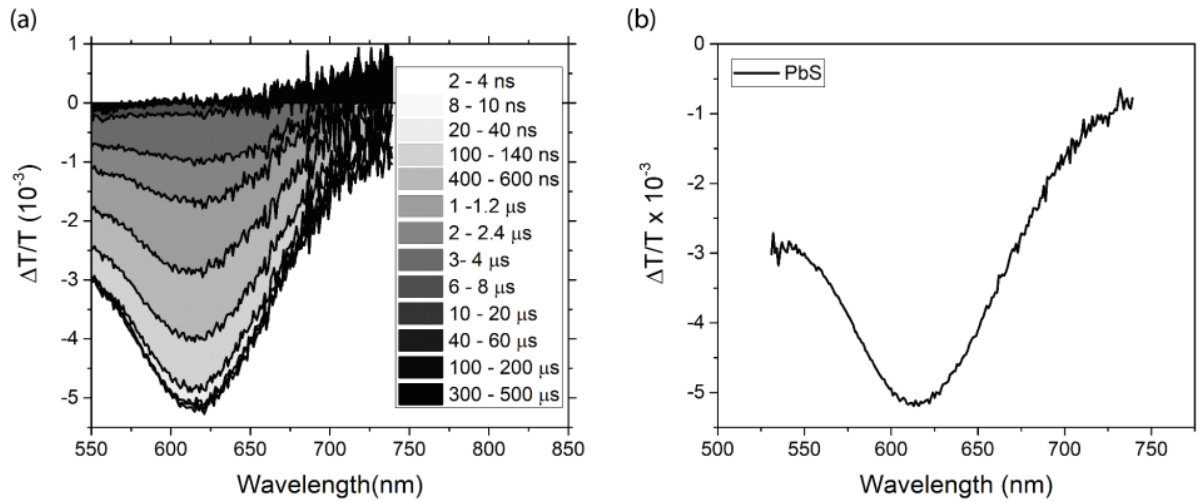

Figure S10: (a) Long-time transient absorption spectra of PbS - OA using $532 \mathrm{~nm}$ excitation $\left(400 \mu \mathrm{J} / \mathrm{cm}^{2}\right)$ (b) DAS of PbS -Tet

Comparing the spectra of the PbS-Tet (TetCAL attached to QDs) with free TetCAL ligand in solution we find good agreement between the spectra at $5 \mathrm{~ns}$ (TT states) and $5 \mu$ s (free triplets) in the region above $650 \mathrm{~nm}$ where the signals from the ligand are strong. This confirms the evolution to free triplets in the PbS-Tet system.

The region below $650 \mathrm{~nm}$ is dominated by signals from $\mathrm{PbS}$, which may be due to charging effects on $\mathrm{PbS}$ (see below). Due to the small quantity of material available we were not able to flow the solutions during this study, which would avoid the charging effect on the PbS. This gives rise to the background signals in the regions below $650 \mathrm{~nm}$. The spectra of the charged dots presents a ground state bleaching feature but not PIAs associated with the excitons. Thus the prominent PIA features peaking at $620 \mathrm{~nm}$ associated with excitons on PbS allows us to track the actual excited states. This feature is clearly observed in the spectra of the pure PbS, figure S10 and also in the PbS-Tet at 50ns, figure $4 \mathrm{~b}$, but not in the spectra at $5 \mu \mathrm{s}$ does not show the. We interpret this as related to the loss of excitations on the $\mathrm{PbS}$ by $5 \mu \mathrm{s}$.

(a)

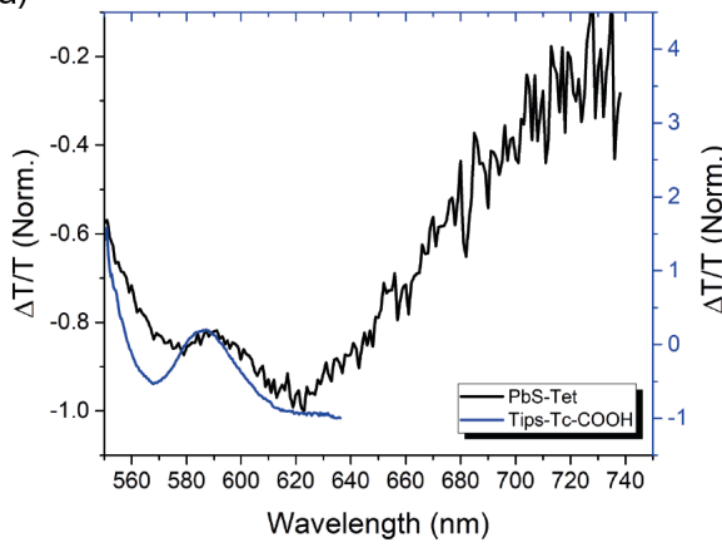

(b)

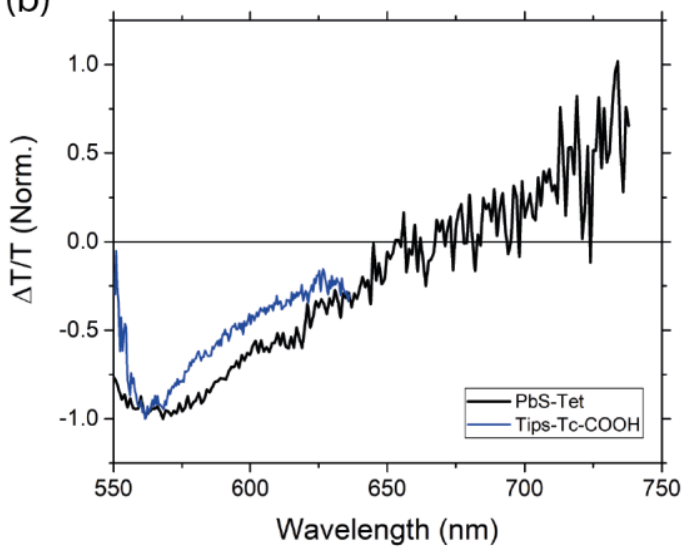

Figure S11: Comparison of the transient absorption spectra of PbS-Tet with free TetCAL ligand in solution at (a) $5 \mathrm{~ns}$ and (b) $5 \mu \mathrm{s}$. 